\section{Saúde bucal de idosos institucionalizados, zona leste de São Paulo, Brasil, 1999}

\author{
Oral health of institutionalized elderly \\ in the Eastern Zone of São Paulo, Brazil, 1999
}

1 Faculdade de Saúde Pública, Universidade de São Paulo, São Paulo, Brasil.

2 Faculdade de Odontologia de Piracicaba, Universidade Estadual de Campinas, Piracicaba, Brasil.

Correspondência M. L. R. Sousa Programa de Pós-graduação em Odontologia, Faculdade de Odontologia de Piracicaba, Universidade Estadual de Campinas. Av. Limeira 901, Piracicaba, $S P$

13414-018, Brasil. luzsousa@fop.unicamp.br

\section{Abstract}

The objective was to verify oral health conditions in institutionalized elderly people in the city of São Paulo, Brazil, through epidemiological analysis. The sample consisted of $293 \mathrm{sub}$ jects and was stratified according to age (65-74 years and over 75) and gender. Epidemiological analysis was conducted according to WHO criteria to determine prevalence of oral disease. Chi-square and Mann-Whitney tests were used to check differences between strata. Of the subjects evaluated, $64.8 \%$ were women and $65.2 \%$ were over 75 years old. DMFT was 30.8 with tooth loss of $96.3 \%$, and this percentage was statistically greater for women (97.0\%); 94.7\% showed calculus as a major degree of periodontal condition, and only $1.8 \%$ presented healthy periodontal conditions and $33.3 \%$ presented loss of attachment ranging from 6 to $8 \mathrm{~mm}$. Some $48.1 \%$ used total upper and $22.5 \%$ total lower prostheses. Edentulism was 68.3\%. The data indicate unsatisfactory clinical conditions, as shown by the high CPO-D index and high percentage of edentulism.

Aged; Health Surveys; Oral Health
Rosane Maria do Valle Carneiro 1 Débora Dias da Silva 2

Maria da Luz Rosário de Sousa 2 Ronaldo Seichi Wada ${ }^{2}$

\section{Introdução}

No Brasil, a Odontologia tem dado seus primeiros passos em direção a terceira idade e começa a haver interesse em se quantificar as condições de saúde bucal dos idosos. Os levantamentos epidemiológicos, que até pouco tempo atrás não contemplavam os indivíduos com mais de sessenta anos, começaram a incluí-los 1,2,3. Entretanto, estes levantamentos foram realizados em idosos na faixa etária preconizada pela OMS (Organização Mundial da Saúde) 4, que inclui indivíduos com idade entre 65 e 74 anos.

Estudos brasileiros relatam como principais alterações bucais encontradas em indivíduos idosos, a cárie dentária, as doenças periodontais, as abrasões, a presença de lesões, o câncer bucal e a perda de grande número de dentes ou o edentulismo 5,6.

Contudo, essas alterações podem variar dependendo se esses idosos estão institucionalizados ou não 7,8,9,10. Normalmente a maioria dos levantamentos são direcionados aos idosos não institucionalizados (usuários de serviços, freqüentadores de grupos de terceira idade ou grêmios), descrevendo dados de saúde bucal insatisfatória. Como existem poucos trabalhos sobre a saúde bucal de idosos institucionalizados $7,8,9$, o enfoque deste estudo foi para esse grupo.

Como exemplos de levantamentos com idosos não-institucionalizados, tem-se os referen- 
tes ao Estado de São Paulo, Brasil, realizados em 1998 1 e $2002^{2}$, com índice CPO-D igual a 28,7 e 28,2 , respectivamente. No Brasil, em 2003 3, o CPO-D foi de 27,8 , sendo que em todos a maior porcentagem do índice correspondeu à perda dentária (em torno de 93,0\%). Outros relatos, ainda referentes a idosos não-institucionalizados, apontam para um maior número de dentes presentes, menor porcentagem de dentes perdidos e conseqüentemente um menor número de indivíduos edêntulos 7,8.

Por outro lado, a saúde bucal de idosos institucionalizados brasileiros apresenta-se com alta porcentagem de edêntulos, variando entre $72,0 \%$ e $84,0 \% 7,8$, entretanto, por vezes, esta porcentagem de edentulismo é numericamente menor $(54,5 \%) 9$.

Quanto às doenças periodontais, estas podem se manifestar desde a forma de uma gengivite até a forma de periodontites severas com conseqüente mobilidade e perda do elemento dentário, podendo levar ao uso e necessidade de próteses. Apesar de comum nos idosos, a doença periodontal não é conseqüência da idade 5 , tornando-se importante estudos sobre esta ocorrência.

Neste contexto de busca pelo conhecimento da real situação em que se encontra a saúde bucal de indivíduos com idade acima de 65 anos, considerou-se importante realizar este trabalho, com o objetivo de quantificar as condições de saúde bucal de idosos institucionalizados na cidade de São Paulo, tendo em vista que os dados divulgados neste Município datam de 19897.

\section{Material e métodos}

A coleta de dados foi realizada entre 27 de setembro e 2 de dezembro de 1999, por uma examinadora, com a utilização de códigos e critérios da OMS 4.

Na época da coleta de dados, existiam 147 instituições para idosos cadastradas no Município de São Paulo, todas de caráter privado, segundo dados fornecidos pela Secretaria de Saúde, divididas entre os cinco Núcleos Regionais de Saúde da Vigilância Sanitária, sendo o Núcleo V selecionado por conveniência para a realização deste estudo.

Esse Núcleo (que inclui a zona leste de São Paulo) possuía 23 instituições, e o critério préestabelecido foi selecionar as de médio e grande porte, totalizando cinco instituições. Foram examinados 293 idosos com 65 anos ou mais, tanto homens quanto mulheres. Optou-se por utilizar duas faixas etárias: 65 a 74 anos, reco- mendada pela OMS, para permitir comparações com dados de outros locais e 75 anos ou mais, pois era esperado grande número de idosos pertencentes a essa faixa etária, em se tratando de asilos.

Segundo critérios preconizados pela OMS 4 e adaptados pela Faculdade de Saúde Pública, Universidade de São Paulo (FSP-USP) 11, foram avaliadas as condições de coroa, sendo que para medir a prevalência da cárie, utilizou-se o índice CPO-D, que indica o número de dentes permanentes cariados, perdidos e restaurados. A condição periodontal foi avaliada com o uso do índice periodontal comunitário (CPI) e de perda de inserção periodontal (PIP), considerando-se o indivíduo. Também foi verificado o uso de próteses totais, bem como o acesso à assistência odontológica, com a pergunta: "possui assistência odontológica?".

Durante a coleta de dados, cerca de 10,0\% dos exames foram realizados em duplicata 4 para verificação da concordância intra-examinadora, utilizando-se a "porcentagem geral de concordância" 12. Dos trinta reexames realizados, foram encontrados resultados dentro dos parâmetros de confiabilidade, classificados como "excelente concordância" (acima de 97,5\% para condição de coroa e uso de prótese) e "ótima concordância” (acima de 95,0\% para o CPI e PIP) 12 . Os valores relatados são também considerados dentro dos parâmetros de confiabilidade da OMS 4.

\section{Coleta de dados}

Os idosos foram examinados nas próprias instituições selecionadas para a realização do estudo, com o uso de espátulas de madeira descartáveis e/ou espelhos bucais e sondas periodontais CPI (tipo ball-point) previamente esterilizados, sendo usado um fotóforo completo (com uso de energia elétrica - marca Missouri) como foco de iluminação artificial, levando-se em consideração que o local onde o indivíduo era examinado não apresentava iluminação natural suficiente para a realização do exame.

\section{Processamento dos dados \\ e análise estatística}

Foi utilizado para análise dos dados o programa Epi Info, versão 5.0, os testes de qui-quadrado (para comparação dos dados porcentuais) e Mann-Whitney (para comparação de médias), com nível de significância de 95\%.

O início da coleta de dados deu-se após a aprovação deste estudo pelo Comitê de Ética em Pesquisa da FSP-USP, com a obtenção do 
termo de consentimento livre e esclarecido assinado pelos voluntários.

\section{Resultados}

Na ocasião da coleta dos dados foi encontrado um total de 400 idosos, dos quais 337 tinham 65 anos de idade ou mais (idade mínima estipulada para este estudo; a idade máxima encontrada foi 97 anos), destes, 293 (86,9\%) participaram da pesquisa. A perda $(13,1 \%)$ ocorreu pela recusa em participar, falta de condições físicas e/ou mentais para a realização do exame, hospitalização e/ou inexistência de documentos para comprovação da idade declarada.

Observou-se um maior número de idosos na faixa etária de 75 e mais $(n=191)$, e com relação ao gênero, a maioria foi de mulheres ( $\mathrm{n}=$ 190). Faz-se aqui uma ressalva à existência de uma instituição que abrigava somente mulheres.

Não havia nenhum dente na arcada superior em $83,6 \%$ da amostra, sendo que esta porcentagem na arcada inferior foi de $73,4 \%$. Dentre todos os indivíduos examinados, $68,3 \%(n=200)$ eram totalmente edêntulos.

Dentre esses edêntulos, $42,0 \%$ não usavam prótese total superior (PTS), 68,5\% não usavam prótese total inferior (PTI), 42,0\% não usavam nem PTS nem PTI, ao passo que apenas 31,5\% usavam ambas. O uso somente de PTS foi encontrado em $26,5 \%$ dos idosos edêntulos, porém, não foi encontrado o uso somente de PTI.

$\mathrm{O}$ índice CPO-D encontrado na amostra foi de 30,8 dentes e o componente "dentes perdidos" correspondeu a 96,3\%; quando foi feita a análise do CPO-D, não houve diferença tanto com relação à faixa etária $(\mathrm{p}=0,22)$ (Tabela 1 ) quanto em relação ao gênero $(p=0,14)$ (Tabela 2).

Quando da análise dos componentes do índice CPO-D nas faixas etárias, verificou-se que não houve diferença entre elas $(p>0,05)$, porém quando esta análise foi realizada por gênero, houve diferença em todos os componentes, onde os homens apresentaram mais dentes com cárie $(p=0,00)$, menos dentes restaurados $(\mathrm{p}=0,01)$ e perdidos $(\mathrm{p}=0,00)$ (Tabela 2$)$.

Com relação aos dentes presentes e hígidos, a amostra como um todo apresentou 2,3 dentes presentes, sendo que destes, 1,2 encontravam-se hígidos. Os indivíduos com idade entre 65 e 74 anos apresentaram mais dentes hígidos quando comparados com aqueles com mais de 75 anos ( $p=0,02$ ) (Tabela 1 ), mas não houve diferença em relação aos dentes presentes $(p>0,05)$. Os homens apresentaram mais dentes presentes e hígidos $(\mathrm{p}=0,00)$ que as mulheres (Tabela 2).

A condição periodontal com maior prevalência (CPI) foi relativa ao cálculo $(94,7 \%)$, sendo que não houve diferença entre as porcentagens de idosos que apresentavam esta condição tanto para a faixa etária, quanto para o gênero $(p=0,84)$. Somente $1,8 \%$ apresentou condição periodontal sadia e o restante $(3,5 \%)$ apresentou bolsas acima de $4 \mathrm{~mm}$ (dados não apresentados).

Quanto ao PIP, desconsiderando os sextantes nulos, a maior porcentagem de idosos apresentou perda entre 6 e $8 \mathrm{~mm}$, seguida de perda entre 0 e $3 \mathrm{~mm}$ para as faixas etárias. Quanto ao gênero, as maiores prevalências foram de perdas entre 6 e $8 \mathrm{~mm}$ para os homens e entre 0 e $3 \mathrm{~mm}$ para as mulheres. Verificou-se que não houve diferença com relação às perdas de inserção

Tabela 1

Índice CPO-D e componentes, dentes presentes e hígidos em idosos institucionalizados, segundo faixa etária. São Paulo, Brasil, 1999.

\begin{tabular}{|c|c|c|c|c|c|c|c|}
\hline \multirow[t]{3}{*}{ Condição } & \multicolumn{6}{|c|}{ Faixa etária (anos) } & \multirow[t]{3}{*}{ Valor de $p$} \\
\hline & \multicolumn{2}{|c|}{$65-74(n=102)$} & \multicolumn{2}{|c|}{$75 e+(n=191)$} & \multicolumn{2}{|c|}{ Total $(n=293)$} & \\
\hline & média & $\%$ & média & $\%$ & média & $\%$ & \\
\hline Dentes perdidos* & 29,5 & 96,3 & 29,7 & 96,3 & 29,6 & 96,3 & 0,93 \\
\hline Dentes cariados* & 0,9 & 3,1 & 0,8 & 2,8 & 0,9 & 2,9 & 0,38 \\
\hline Dentes restaurados* & 0,2 & 0,6 & 0,3 & 0,9 & 0,2 & 0,8 & 0,13 \\
\hline CPO-D** & 30,6 & & 30,8 & & 30,8 & & 0,22 \\
\hline Dentes presentes* & 2,5 & & 2,2 & & 2,3 & & 0,09 \\
\hline Dentes hígidos* & 1,4 & & 1,1 & & 1,2 & & 0,02 \\
\hline
\end{tabular}

* Teste qui-quadrado (comparação de percentuais com base no número de dentes);

** Teste Mann-Whitney (comparação de médias: CPO-D). 
Índice CPO-D e componentes, dentes presentes e hígidos em idosos institucionalizados, segundo gênero. São Paulo, Brasil, 1999.

\begin{tabular}{|c|c|c|c|c|c|c|c|}
\hline \multirow[t]{2}{*}{ Condição } & \multicolumn{6}{|c|}{ Gênero } & \multirow[t]{2}{*}{ Valor de $p$} \\
\hline & Média & $\%$ & Média & $\%$ & Média & $\%$ & \\
\hline Dentes perdidos* & 30,1 & 96,9 & 28,7 & 95,0 & 29,6 & 96,3 & 0,00 \\
\hline Dentes cariados* & 0,6 & 2,1 & 1,4 & 4,5 & 0,9 & 2,9 & 0,00 \\
\hline Dentes restaurados* & 0,3 & 0,1 & 0,1 & 0,5 & 0,2 & 0,8 & 0,01 \\
\hline CPO-D** & 31,1 & & 30,2 & & 30,8 & & 0,14 \\
\hline Dentes presentes* & 1,9 & & 3,2 & & 2,3 & & 0,00 \\
\hline Dentes hígidos* & 0,9 & & 1,7 & & 1,2 & & 0,00 \\
\hline
\end{tabular}

* Teste qui-quadrado (comparação de percentuais com base no número de dentes);

** Teste Mann-Whitney (comparação de médias: CPO-D).

periodontal tanto para a faixa etária $(\mathrm{p}>0,05)$ (Tabela 3), quanto para o gênero (p >0,05) (Tabela 4).

Com relação ao uso de próteses, houve um número inexpressivo de uso de outras próteses que não a total, assim, os dados apresentados se referem à prótese total. Para a arcada superior (PTS), 48,12\% usavam prótese total e para a inferior (PTI), a porcentagem foi de $22,53 \%$ (dados não apresentados).

No geral, 59,5\% dos indivíduos não possuíam assistência odontológica, esta porcentagem foi de $56,9 \%$ na faixa etária dos 65 aos 74 anos e de $63,4 \%$ na de 75 anos e mais. Com relação ao gênero, 55,8\% das mulheres e 70,9\% dos homens, não possuíam assistência (dados não apresentados).

\section{Discussão}

Conforme os resultados de erro intra-examinador, pode-se afirmar que o estudo apresentou confiabilidade, como recomendado pela OMS 4.

Convém ressaltar que neste estudo as instituições às quais esses idosos pertenciam eram particulares, mas de cunho filantrópico, com uma pequena parcela de abrigados pagantes (baixos valores). Esse fato provavelmente caracterizou a população estudada como oriunda de classes sociais mais baixas, porém, homogênea entre si. Nesta amostra conseguiu-se número suficiente de mulheres e homens, possibilitando abordar este tema, e assim, os dados devem ser verificados sob esta óptica.

O levantamento epidemiológico mais recente sobre saúde bucal no Brasil teve uma abordagem domiciliar 3; outros estudos epidemiológicos 1,8,13 abordaram idosos oriundos de grêmios, de grupos de terceira idade ou usuários de serviços de saúde. Assim, o presente estudo buscou identificar se os resultados sobre saúde bucal de idosos institucionalizados iriam apresentar-se de forma diferente, mesmo conhecendo-se previamente pela literatura que os idosos apresentam altos porcentuais de perda dentária.

Neste estudo, os idosos institucionalizados apresentaram uma alta porcentagem de dentes perdidos (96,3\%), fato que fez com que elevasse a média CPO-D $(30,8)$. Comparando-se com dados dos levantamentos conduzidos no Brasil três anos após o presente estudo ${ }^{3}$, o CPO-D foi de 27,8 tendo 93,0\% de dentes perdidos. Para o Estado de São Paulo 1, realizado aproximadamente na mesma época deste estudo com idosos não-institucionalizados, o CPO-D foi de 28,7 , com 93,0\% de dentes perdidos. Verifica-se que os dados de saúde bucal de idosos institucionalizados apresentam-se em piores condições do que aqueles em grupos não-institucionalizados.

Nesse levantamento do Estado de São Paulo 1, o CPO-D para a faixa etária de 65 a 74 anos foi de 28,7 e, na DIR I (Direção Regional de Saúde na qual São Paulo está inserida) 1 foi de 28,2, estando também abaixo do encontrado neste estudo $(30,8)$, outros dados que revelam a diferença (mesmo que numérica) existente entre idosos institucionalizados e não-institucionalizados.

Em idosos institucionalizados com idade acima de sessenta anos, Rosa et al. 7 verificaram na cidade de São Paulo, o índice CPO-D $(30,97)$ e porcentagem de dentes perdidos $(96,1 \%)$ com valores tão elevados quanto aos do presente estudo, além de maior porcentagem de edentulismo $(84,0 \%)$. Pode-se observar que o pa- 
Número e porcentagem de idosos institucionalizados, segundo a perda de inserção periodontal (PIP) e faixa etária. São Paulo, Brasil, 1999.

\begin{tabular}{|c|c|c|c|c|c|c|c|}
\hline \multirow[t]{3}{*}{ Condição } & \multicolumn{4}{|c|}{ Faixa etária (anos) } & \multicolumn{2}{|c|}{ Total } & \multirow[t]{3}{*}{ Valor de $p$} \\
\hline & \multicolumn{2}{|c|}{$65-74$} & \multicolumn{2}{|c|}{$75 e+$} & \multirow[b]{2}{*}{$n$} & \multirow[b]{2}{*}{$\%$} & \\
\hline & $\mathrm{n}$ & $\%$ & $\mathrm{n}$ & $\%$ & & & \\
\hline $0(0-3 \mathrm{~mm})^{\star}$ & 6 & 27,3 & 9 & 25,7 & 15 & 26,3 & 0,89 \\
\hline $1(4-5 \mathrm{~mm})^{\star}$ & - & - & - & - & - & - & - \\
\hline $2(6-8 m m)^{*}$ & 8 & 36,4 & 11 & 31,4 & 19 & 33,4 & 0,70 \\
\hline $3(9-11 \mathrm{~mm})^{\star}$ & 3 & 13,6 & 7 & 20,0 & 10 & 17,5 & 0,53 \\
\hline $4(12 \mathrm{~mm} \text { ou }+)^{*}$ & 5 & 22,7 & 8 & 22,9 & 13 & 22,8 & 0,99 \\
\hline Total & 22 & 100,0 & 35 & 100,0 & 57 & 100,0 & \\
\hline
\end{tabular}

* Teste qui-quadrado.

Número e porcentagem de idosos institucionalizados, segundo a perda de inserção periodontal (PIP) e gênero. São Paulo, Brasil, 1999.

\begin{tabular}{|c|c|c|c|c|c|c|c|}
\hline \multirow[t]{3}{*}{ Condição } & \multicolumn{4}{|c|}{ Gênero } & \multicolumn{2}{|c|}{ Total } & \multirow[t]{3}{*}{ Valor de $p$} \\
\hline & \multicolumn{2}{|c|}{ Mulheres } & \multicolumn{2}{|c|}{ Homens } & & & \\
\hline & $\mathrm{n}$ & $\%$ & $n$ & $\%$ & $\mathrm{n}$ & $\%$ & \\
\hline $0(0-3 \mathrm{~mm})^{\star}$ & 12 & 34,4 & 3 & 13,6 & 15 & 26,3 & 0,08 \\
\hline $1(4-5 \mathrm{~mm})^{\star}$ & - & - & - & - & - & - & - \\
\hline $2(6-8 \mathrm{~mm})^{*}$ & 11 & 31,4 & 8 & 36,4 & 19 & 33,4 & 0,70 \\
\hline $3(9-11 \mathrm{~mm})^{\star}$ & 6 & 17,1 & 4 & 18,2 & 10 & 17,5 & 0,92 \\
\hline $4(12 \mathrm{~mm} \text { ou }+)^{*}$ & 6 & 17,1 & 7 & 31,8 & 13 & 22,8 & 0,19 \\
\hline Total & 35 & 100,0 & 22 & 100,0 & 57 & 100,0 & \\
\hline
\end{tabular}

* Teste qui-quadrado.

drão de resultados tem-se mantido inalterado, mesmo dez anos após a realização daquele estudo, visto que foi realizado em 1989.

Ainda foram encontradas em idosos institucionalizados com idade acima de sessenta anos, na cidade de Araraquara, São Paulo, por Silva \& Valsecki Jr. 8, CPO-D 30,91 e 72,0\% de edentulismo, condições muito próximas às do presente estudo. Já Martins Neto \& Padilha 9, no Rio Grande do Sul, relataram CPO-D de 31,05 , valor próximo ao do presente estudo; no entanto, a porcentagem de edentulismo foi bem menor $(54,5 \%)$.

Por outro lado, num estudo longitudinal realizado em uma instituição para idosos (67 a 102 anos) na Noruega, em 1980 e 1993, Jokstad et al. 14 obtiveram CPO-D de 25,7 e 22,9, respectivamente, médias bem menores que as encontradas nos estudos discutidos anterior- mente e no presente estudo, mesmo há seis anos atrás.

Com relação ao percentual de dentes perdidos no presente estudo, foi verificada diferença estatística somente quando da análise por gênero, onde as mulheres obtiveram uma porcentagem maior que os homens. Isto talvez se deva ao fato de as mulheres se preocuparem mais com a sua saúde e, conseqüentemente, procurarem assistência 15 , que no caso, foi predominantemente de cunho mutilador. Ressalta-se que no presente estudo houve maior porcentagem de mulheres com assistência odontológica do que homens.

Por outro lado, Cardoso et al. 16 observaram em sua população de estudo, com idade entre 65 e 84 anos, que quanto menor era o poder aquisitivo, maiores eram as necessidades de assistência odontológica (piores condições 
bucais), fato que caracteriza a concordância com os resultados do presente estudo, ou seja, a maioria dos idosos não possuía assistência odontológica.

Quanto à média de dentes presentes e hígidos, no trabalho de Rosa et al. 7, os idosos institucionalizados apresentaram em média 2,0 dentes presentes e 1,0 hígido. Silva \& Valsecki Jr. 8 encontraram 3,15 dentes presentes, sendo 1,09 hígido, valores muito próximos aos encontrados no presente estudo (2,3 presentes e 1,2 hígido).

No Estado de São Paulo 1 foram registrados 2,65 dentes hígidos por idoso, estando, portanto, acima do presente estudo, que também obteve a média de dentes presentes abaixo das encontradas no Estado de São Paulo ${ }^{1}(4,68)$ e na DIR I $1(5,20)$. Também por meio desses dados podem-se verificar piores condições de saúde bucal para os idosos institucionalizados, tendo em vista que esses levantamentos citados para comparação ${ }^{1}$ não se referem a idosos institucionalizados.

Slade et al. 17 compararam condições de saúde bucal em idosos institucionalizados e não-institucionalizados em Ontário, Canadá, concluindo que os idosos institucionalizados têm duas vezes mais chances de serem desdentados.

Bourgeois et al. 18 apresentaram dados sobre edentulismo em idosos com idade entre 65 e 74 anos, avaliados em 21 países, sendo o mais alto percentual encontrado na Islândia, com 69,6\% de edêntulos (1992). A Holanda segue logo atrás com $65,4 \%$ (1986) e a Dinamarca com $60,0 \%$ (1987). Em 5, do total dos 21 países, o edentulismo esteve presente em mais de 50,0\% da população; além dos três citados, soma-se o Reino Unido com $57,0 \%$ (1988) e a Hungria com $53,3 \%$ (1991). Os países onde foram encontradas menores porcentagens de indivíduos edêntulos foram: a Itália, com apenas $12,8 \%$ (1993), a Áustria com 14,9\% (1992), a Suíça com 15,3\% (1988) e a França com 16,3\% (1995). Angelillo et al. 19, numa amostra de 234 idosos institucionalizados na Itália, encontraram 140 desdentados totais $(59,8 \%)$.

Com esses dados, verifica-se que o edentulismo é ainda um dos grandes problemas de saúde pública em diferentes localidades e épocas, com grande variação $(13,0 \%$ a $70,0 \%)$, sendo que os dados apresentados atualmente no Brasil são de alta porcentagem de edentulismo.

Comparando o presente estudo com outros estudos nacionais com idosos institucionalizados, percebe-se o elevado índice CPO-D, alta porcentagem de dentes perdidos e conseqüentemente um grande número de indivíduos edêntulos, conseqüência não só da cárie, mas também das doenças periodontais, evidenciadas neste estudo pelo baixo percentual de indivíduos com sextantes válidos para a avaliação periodontal, e esses indivíduos, apesar de apresentarem cálculo como maior prevalência do CPI, 33,0\% já apresentavam bolsas entre 6 e $8 \mathrm{~mm}$ como maior prevalência para o PIP, o que indica a grande possibilidade de se partir destas condições para o uso de próteses.

Verificou-se que as próteses usadas eram predominantemente as totais, devido ao alto número de edêntulos. Entre as próteses totais utilizadas, tem-se que mais indivíduos usavam as superiores que as inferiores. Dentre os edêntulos, uma grande parte $(42,0 \%)$ não usava nem superior, nem inferior, gerando impactos negativos tanto na função mastigatória quanto na fonética, além da deglutição e estética, fatores estes que implicam diretamente a qualidade de vida e bem-estar desses indivíduos.

Ressalta-se que uma das metas estabelecidas para o ano 2000, como desejável para a idade de 65 anos ou mais, era a redução de 25,0\% dos níveis observados de edentulismo e a presença de vinte ou mais dentes funcionais em mais de $50,0 \%$ dos indivíduos 20 . O presente estudo mostra dados bem aquém destas metas.

Pucca Jr. 21 em estudo realizado com idosos de 65 anos de idade ou mais, no Município de São Paulo, obteve prevalência auto-referida de edentulismo de $54,9 \%$ no primeiro inquérito domiciliar e de $56,0 \%$ no segundo, porcentagens que mesmo consideradas altas, encontram-se abaixo da relatada no presente estudo $(68,3 \%)$. Acrescenta-se a este fato que quando há referência de que o idoso apresenta falta de dentes, isto representa a falta de todos os dentes. Só para se ter idéia, a cada quatro brasileiros com mais de sessenta anos, três não têm dentes em pelo menos uma das arcadas, o que corresponde a $75,0 \% 22$.

Segundo Terarolli Jr. et al. 23 , o rápido envelhecimento da população é uma realidade, e assim, torna-se imprescindível a adoção de políticas sociais que possam suprir as necessidades relativas à assistência social e de saúde, para garantir aos idosos uma melhor qualidade aos anos de vida, que têm aumentado nas últimas décadas, com o aumento da expectativa de vida. Com relação à saúde bucal dos idosos, o edentulismo parece ser "natural", entretanto, este quadro pode ser reflexo de uma política preventiva deficiente, que deveria ser direcionada a todos os grupos etários 24 . 


\section{Conclusões}

Esse grupo de idosos institucionalizados pode ser caracterizado com condições clínicas insatisfatórias, pois o número de dentes perdidos registrado foi alto, evidenciado pelo grande percentual de edêntulos. Dentre os totalmente edêntulos, 42,0\% não usavam próteses totais (nem superiores, nem inferiores), denotando que a sociabilização desses indivíduos pode estar comprometida, pois os dentes afetam a mastigação, fonação, deglutição e também a estética, o que irá interferir na qualidade de vida.

Ressalta-se a importância da epidemiologia como um instrumento útil e que, portanto deve ser usado no planejamento de serviços em odontologia, com prioridade frente às necessidades encontradas, além de possibilitar o estudo das tendências das condições de saúde bucal no grupo de idosos.

\section{Resumo}

Verificar as condições de saúde bucal de idosos institucionalizados, na cidade de São Paulo, Brasil, por meio de exame epidemiológico. Foram selecionados 293 indivíduos, estratificados segundo a faixa etária (65-74 anos e 75 anos e mais) e gênero. O exame epidemiológico foi realizado segundo critérios preconizados pela Organização Mundial da Saúde para determinar a prevalência das doenças bucais. Foram usados os testes qui-quadrado e Mann-Whitney para verificar as diferenças entre as estratificações com nível de significância de 95\%. Do total dos indivíduos, 64,8\% eram mulheres e 65,2\% tinham 75 anos ou mais. O CPO-D foi de 30,8, com 96,3\% de dentes perdidos, sendo que esta porcentagem foi estatisticamente maior para as mulheres (97,0\%); 94,7\% apresentaram cálculo como maior grau de condição periodontal; apenas 1,8\% possuía condição periodontal sadia e 33,3\% tiveram perdas de inserção entre 6 e $8 \mathrm{~mm}$. Quanto ao uso de prótese total, 48,1\% usavam prótese superior e 22,5\% inferior. A porcentagem de indivíduos totalmente edêntulos foi de 68,3\%. Os dados apresentados caracterizaram condições clínicas insatisfatórias, podendo ser evidenciadas pelo elevado índice CPO-D e alta porcentagem de edentulismo.

Idoso; Levantamentos Epidemiológicos; Saúde Bucal

\section{Colaboradores}

R. M. V. Carneiro participou do planejamento inicial do estudo, realizou a coleta de dados, interpretou os resultados e iniciou a redação do texto. D. D. Silva finalizou a redação e revisão do texto. M. L. R. Sousa colaborou com a orientação durante a interpretação dos resultados, bem como na redação e revisão do texto. R. S. Wada analisou os dados e interpretou os resultados.

\section{Agradecimentos}

Ao Conselho Nacional de Desenvolvimento Científico e Tecnológico (CNPq) pela bolsa concedida, que viabilizou a realização deste estudo. 


\section{Referências}

1. Secretaria de Estado da Saúde de São Paulo/Núcleo de Estudos e Pesquisas de Sistemas de Saúde, Faculdade de Saúde Pública, Universidade de São Paulo. Levantamento epidemiológico em saúde bucal: Estado de São Paulo, 1998. São Paulo: Faculdade de Saúde Pública, Universidade de São Paulo; 1999.

2. Secretaria de Estado da Saúde de São Paulo/Centro Técnico de Saúde Bucal, Núcleo de Estudos e Pesquisas de Sistemas de Saúde, Faculdade de Saúde Pública, Universidade de São Paulo. Condições de saúde bucal no Estado de São Paulo em 2002. São Paulo: Faculdade de Saúde Pública, Universidade de São Paulo; 2002.

3. Ministério da Saúde. Projeto SB Brasil 2003: condições de saúde bucal da população brasileira 2002-2003. Resultados principais. Brasília: Ministério da Saúde; 2004.

4. World Health Organization. Oral health surveys: basic methods. $4^{\text {th }}$ Ed. Geneva: World Health Organization; 1997.

5. Silva SRC. Autopercepção das condições bucais em pessoas com 60 anos e mais de idade [Tese de Doutorado]. São Paulo: Faculdade de Saúde Pública, Universidade de São Paulo; 1999.

6. Pucca Jr. GA. Saúde bucal do idoso: aspectos sociais e preventivos. In: Papaléo Netto M, organizador. Gerontologia. São Paulo: Editora Atheneu; 2002. p. 297-310.

7. Rosa AGF, Fernandez RAC, Pinto VG, Ramos LR. Condições de saúde bucal em pessoas de 60 anos ou mais no Município de São Paulo. Rev Saúde Pública 1992; 26:155-60.

8. Silva SRC, Valsecki Jr. A. Avaliação das condições de saúde bucal dos idosos em um município brasileiro. Rev Panam Salud Pública 2000; 8:268-71.

9. Martins Neto M, Padilha DMP. Avaliação clínica da saúde bucal e do uso do fumo de três grupos de idosos. Rev Odonto Ciênc 2000; 31:53-70.

10. McMillan AS, Wong MCM, Lo ECM, Allen PF. The impact of oral disease among the institutionalized and non-institutionalized elderly in Hong Kong. J Oral Rehabil 2003; 30:46-54.

11. Secretaria de Estado da Saúde de São Paulo/Núcleo de Estudos e Pesquisas de Sistemas de Saúde, Faculdade de Saúde Pública, Universidade de São Paulo. Levantamento epidemiológico em saúde bucal: Estado de São Paulo, 1998 - caderno de instruções. São Paulo: Faculdade de Saúde Pública, Universidade de São Paulo; 1998.
12. Frias AC. Estudo de confiabilidade do levantamento epidemiológico de saúde bucal. Estado de São Paulo, 1998 [Dissertação de Mestrado]. São Paulo: Faculdade de Saúde Pública, Universidade de São Paulo; 2000.

13. Silva DD, Sousa MLR, Wada RS. Saúde bucal em adultos e idosos de Rio Claro, São Paulo, Brasil. Cad Saúde Pública 2004; 20:626-31.

14. Jokstad A, Ambjornsen E, Eide KE. Oral health in institutionalized elderly people in 1993 compared with in 1980. Acta Odontol Scand 1996; 54:303-8.

15. Schoueri Jr. R, Ramos LR, Papaléo Netto M. Crescimento populacional: aspectos demográficos e sociais. In: Carvalho Filho ET, Papaléo Netto M, organizadores. Geriatria: fundamentos, clínica e terapêutica. São Paulo: Editora Atheneu; 1994. p. 9-29.

16. Cardoso LM, Consalter CE, Domingues F, Lançoni D, Massi FHP, Souza AL, et al. Odontogeriatria: uma nova perspectiva na odontologia. Semina 1999; 20:28-33.

17. Slade GD, Locker D, Leake JL, Price AS, Chao I. Differences in oral health status between institutionalized and non-institutionalized older adults. Community Dent Oral Epidemiol 1990; 18:272-6.

18. Bourgeois D, Nihtila A, Mersel A. Prevalence of caries and edentulousness among 65-74-yearolds in Europe. Bull World Health Organ 1998; 76:413-7.

19. Angelillo IF, Sagliocco G, Hendricks SJH, Villari P. Tooth loss and dental caries in institutionalized elderly in Italy. Community Dent Oral Epidemiol 1990; 18:216-8.

20. Federatión Dentaire Internationale. Global goals for oral health in 2000. Int Dent J 1982; 32:74-7.

21. Pucca Jr. GA. A saúde bucal do idoso: aspectos demográficos e epidemiológicos. http://www. odontologia.com.br/artigos/geriatria.html (acessado em 20/Nov/2000).

22. Ministério da Saúde. Orientações para implantação de equipes de saúde bucal. Brasília: Ministério da Saúde; 2003.

23. Telarolli Jr. R, Machado JCMS, Carvalho F. Perfil demográfico e condições sanitárias dos idosos em área urbana do Sudeste do Brasil. Rev Saúde Pública 1996; 30:485-98.

24. Colussi CF, Freitas SFT. Aspectos epidemiológicos da saúde bucal do idoso no Brasil. Cad Saúde Pública 2002; 18:1313-20.

Recebido em 03/Nov/2004

Versão final reapresentada em 01/Mar/2005

Aprovado em 04/Mai/2005 